\title{
The gauge transformation of the modified KP hierarchy
}

Jipeng Cheng

To cite this article: Jipeng Cheng (2018) The gauge transformation of the modified KP hierarchy, Journal of Nonlinear Mathematical Physics 25:1, 66-85, DOI: https://doi.org/10.1080/14029251.2018.1440743

To link to this article: https://doi.org/10.1080/14029251.2018.1440743

Published online: 04 January 2021 


\title{
The gauge transformation of the modified KP hierarchy
}

\author{
Jipeng Cheng \\ Department of Mathematics, China University of Mining and Technology \\ Xuzhou, Jiangsu 221116, P. R. China \\ Email: chengjp@cumt.edu.cn and chengjp@mail.ustc.edu.cn
}

Received 9 June 2017

Accepted 19 August 2017

\begin{abstract}
In this paper, we firstly investigate the successive applications of three elementary gauge transformation operators $T_{i}$ with $i=1,2,3$ for the mKP hierarchy in Kupershmidt-Kiso version, and find that the gauge transformation operators $T_{i}$ can not commute with each other. Then two types of gauge transformation operators $T_{D}$ and $T_{I}$ constructed from $T_{i}$ are proved that they can commute with each other. In particular, $T_{I}$ is introduced for the first time in the literature. And the successive applications of $T_{D}$ and $T_{I}$ in the form of $T^{(n, k)}$, which is the product of $n$ terms of $T_{D}$ and $k$ terms of $T_{I}$, are derived in three cases for different $n$ and $k$. At last, the corresponding successive applications of $T_{D}$ and $T_{I}$ on the eigenfunction $\Phi$, the adjoint eigenfunction $\Psi$ and the tau functions $\tau_{0}$ and $\tau_{1}$ are considered.
\end{abstract}

Keywords: mKP hierarchy; gauge transformation; tau function.

2010 Mathematics Subject Classification: 35Q51, 37K10, 37K40

\section{Introduction}

The modified Kadomtsev-Petviashvili (mKP) hierarchy is a system of nonlinear differential equations satisfied by the tau functions introduced in the early 1980s in $[14,15]$. There are several versions of the Lax representations for mKP hierarchy. All the definitions are trying to transfer the relationship between $\mathrm{KdV}$ and $\mathrm{mKdV}$ to the KP situation. The first one is known as the nonstandard integrable system, developed by B. A. Kupershmidt and K. Kiso [16-18, 26], which is defined through the modification of the KP hierarchy ("standard case") $\partial_{t_{n}} L=\left[\left(L^{n}\right)_{\geq 0}, L\right]$ to $\partial_{t_{n}} L=\left[\left(L^{n}\right)_{\geq 1}, L\right]$ (see Section 2 for more details). The second one is defined by the factorization of the Lax operator of the KP hierarchy $[6,19]$. The last one has the representation in terms of differential-difference equations $[8,31,32]$. In this paper, we only consider the first one, i.e., Kupershmidt-Kiso version, since it is comparatively easier to discuss the gauge transformation in the first version than other ones. The mKP hierarchy in Kupershmidt-Kiso version is the particular case of the coupled modified KP hierarchy [32], which are proved that there are two tau functions $\tau_{0}$ and $\tau_{1}$. These two tau functions bring much difficulty in the study of the mKP hierarchy, compared with the KP case [7] only owning a single tau function.

The gauge transformation [2, 26, 27], also called Darboux transformation, provides a simple way to construct solutions for integrable hierarchies. By now, the gauge transformations of many integrable hierarchies have been studied, for example, the KP and $\mathrm{mKP}$ hierarchies $[1-3,9,26,27,29,30,34]$, the BKP and CKP hierarchies [4,10,12,25], the discrete KP and modified discrete KP hierarchies [13,20,21,24,28], the $q$-KP and modified $q$-KP hierarchies $[5,11,22,23,33]$ and so on. Here in this paper, we will investigate the gauge transformation of the mKP hierarchy 
in Kupershmidt-Kiso version. There are three elementary gauge transformation operators $T_{i}$ with $i=1,2,3$ (see Section 3 for more details) for the mKP hierarchy $[26,29,30]$. Here we find that $T_{i}$ with $i=1,2,3$ can not commute with each other and therefore they are inconvenient in the application. Thus two types of gauge transformation operators $T_{D}$ [26] and $T_{I}$ constructed from $T_{i}$ (see Section 4 for more details) are introduced, which are proved that they can commute with each other. And in particular, as far as I know, $T_{I}$ is introduced for the first time in the literature. These gauge transformation operators can not only construct various solutions, but also provide the understanding of the inner structures of the mKP hierarchy, for example, the explicit forms of the tau functions for the mKP hierarchy in Kupershmidt-Kiso version. The main content of this paper is devoted to the successive applications of $T_{D}$ and $T_{I}$.

In the KP case, there are two elementary gauge transformation operators, denoted by $T_{d}$ and $T_{i}$. As for the successive applications of $n$ terms of $T_{d}$ and $k$ terms of $T_{i}$, it is enough to only discuss two cases of $n>k$ and $n=k$ in the KP hierarchy [9], since the case $k>n$ can be derived from the commutativity of $T_{d}$ and $T_{i}$, and also the fact that the inverse of the conjugation of $T_{d}$ is $T_{i}$ and vice versa. However in the mKP case, the inverse of the conjugation of $T_{D}$ is not equal to $T_{I}$ and vice versa. Therefore in the mKP case, if we denote the product of $n$ terms of $T_{D}$ and $k$ terms of $T_{I}$ as $T^{(n, k)}$, all three cases for different $n$ and $k$ should be considered in the successive applications of $T_{D}$ and $T_{I}$. Thus the derivation of $T^{(n, k)}$ in the case of $k>n$ can not be avoided, which is one of the major differences from the corresponding results in the KP hierarchy. In addition, we give the detailed derivations of the assumption of the form of $T^{(n, k)}$. But just knowing the assumed form of $T^{(n, k)}$ is not enough in the derivations of explicit form of $T^{(n, k)}$. Actually, besides the similar conditions of $T_{D}$ and $T_{I}$ (see (4.3)) to the KP case, the additional conditions (see (4.4)) are very crucial in this paper, which is another difference from those in the KP case. At last, the corresponding successive applications of $T_{D}$ and $T_{I}$ on the eigenfunction $\Phi$, the adjoint eigenfunction $\Psi$ and the tau functions $\tau_{0}$ and $\tau_{1}$ are considered. And some examples of $\tau_{0}$ and $\tau_{1}$ are given.

This paper is organized in the following way. In Section 2, some basic facts about the mKP hierarchy are introduced. The successive applications and the commutativity of the elementary gauge transformation operators $T_{i}$ with $i=1,2,3$ are discussed in Section 3. Then in Section 4, the successive applications of the gauge transformation operators $T_{D}(\Phi)$ and $T_{I}(\Psi)$ are investigated in three cases for different $n$ and $k$. Further the application on the (adjoint) eigenfunctions and the tau functions are obtained in Section 5. At last in Section 6, some conclusions and discussions are given.

\section{2. the mKP hierarchy}

The mKP hierarchy in Kupershmidt-Kiso version $[16-18,26]$ is defined as the following Lax equation

$$
\partial_{t_{n}} L=\left[\left(L^{n}\right)_{\geq 1}, L\right], n=1,2,3, \cdots
$$

with the Lax operator $L$ given by the pseudo-differential operator below

$$
L=\partial+u_{0}+u_{1} \partial^{-1}+u_{2} \partial^{-2}+u_{3} \partial^{-3}+\cdots,
$$

Here $\partial=\partial_{x}$ and $u_{i}=u_{i}\left(t_{1}=x, t_{2}, \cdots\right)$. The algebraic multiplication of $\partial^{i}$ with the multiplication operator $f$ is given by the usual Leibnitz rule

$$
\partial^{i} f=\sum_{j \geq 0}\left(\begin{array}{l}
i \\
j
\end{array}\right) f^{(j)} \partial^{i-j}, \quad i \in \mathbb{Z}
$$


where $f^{(j)}=\frac{\partial^{j} f}{\partial x^{j}}$. For $A=\sum_{i} a_{i} \partial^{i}, A_{\geq k}=\sum_{i \geq k} a_{i} \partial^{i}$ and $A_{<k}=\sum_{i<k} a_{i} \partial^{i}$. The name of the mKP hierarchy comes from the fact that (2.1) contains the mKP equation

$$
4 u_{t x}=\left(u_{x x x}-6 u^{2} u_{x}\right)_{x}+3 u_{y y}+6 u_{x} u_{y}+6 u_{x x} \int u_{y} d x
$$

In this paper, for any (pseudo-) differential operator $A$ and a function $f$, the symbol $A(f)$ will indicate the action of $A$ on $f$, whereas the symbol $A f$ will denote just operator product of $A$ and $f$, and $*$ stands for the conjugate operation: $(A B)^{*}=B^{*} A^{*}, \partial^{*}=-\partial, f^{*}=f$. Note that there is the term $u_{0}$ in the Lax operator $L$ (see (2.2)) of the mKP hierarchy, which is different from the case of the KP hierarchy.

Similar to the case of the KP hierarchy, the Lax operator $L$ for the mKP hierarchy can be expressed in terms of the dressing operator $Z$,

$$
L_{m K P}=Z \partial Z^{-1},
$$

where $Z$ is given by

$$
Z=z_{0}+z_{1} \partial^{-1}+z_{2} \partial^{-2}+\cdots\left(z_{0}^{-1} \text { exists }\right)
$$

Then the Lax equation (2.1) is equivalent to

$$
\partial_{t_{n}} Z=-\left(L_{m K P}^{n}\right)_{\leq 0} Z=-\left(Z \partial^{n} Z^{-1}\right)_{\leq 0} Z
$$

Define the wave and the adjoint wave functions of the mKP hierarchy in the following way:

$$
\begin{aligned}
w(t, \lambda) & =Z\left(e^{\xi(t, \lambda)}\right)=\hat{w}(t, \lambda) e^{\xi(t, \lambda)}, \\
w^{*}(t, \lambda) & =\left(Z^{-1} \partial^{-1}\right)^{*}\left(e^{-\xi(t, \lambda)}\right)=\hat{w}^{*}(t, \lambda) \lambda^{-1} e^{-\xi(t, \lambda)},
\end{aligned}
$$

with

$$
\begin{aligned}
\xi(t, \lambda) & =x \lambda+t_{2} \lambda^{2}+t_{3} \lambda^{3}+\cdots \\
\hat{w}(t, \lambda) & =z_{0}+z_{1} \lambda^{-1}+z_{2} \lambda^{-2}+\cdots \\
\hat{w}^{*}(t, \lambda) & =z_{0}^{-1}+z_{1}^{*} \lambda^{-1}+z_{2}^{*} \lambda^{-2}+\cdots
\end{aligned}
$$

Then $w(t, \lambda)$ and $w^{*}(t, \lambda)$ satisfy the bilinear identity [32] below

$$
\operatorname{res}_{\lambda} w\left(t^{\prime}, \lambda\right) w^{*}(t, \lambda)=1
$$

which is equivalent to the mKP hierarchy. Here $\operatorname{res}_{\lambda} \sum_{i} a_{i} \lambda^{i}=a_{-1}$. 
It is proved in [32] that there exist two tau functions ${ }^{\mathrm{a}} \tau_{1}$ and $\tau_{0}$ for the mKP hierarchy in Kupershmidt-Kiso version such that

$$
\begin{aligned}
w(t, \lambda) & =\frac{\tau_{0}\left(t-\left[\lambda^{-1}\right]\right)}{\tau_{1}(t)} e^{\xi(t, \lambda)}, \\
w^{*}(t, \lambda) & =\frac{\tau_{1}\left(t+\left[\lambda^{-1}\right]\right)}{\tau_{0}(t)} \lambda^{-1} e^{-\xi(t, \lambda)} .
\end{aligned}
$$

By comparing (2.11) with (2.14), one can find

$$
z_{0}=\frac{\tau_{0}(t)}{\tau_{1}(t)}, \frac{z_{1}}{z_{0}}=-\partial_{x} \ln \tau_{0}(t)
$$

The eigenfunction $\Phi$ and the adjoint eigenfunction $\Psi$ of the mKP hierarchy are defined in the identities below,

$$
\Phi_{t_{n}}=\left(L^{n}\right)_{\geq 1}(\Phi), \quad \Psi_{t_{n}}=-\left(L^{n}\right)_{\geq 1}^{*}(\Psi)
$$

\section{Elementary Gauge Transformation}

In this section, we will investigate the elementary gauge transformation of the mKP hierarchy. For the mKP hierarchy (2.1), suppose $T$ is a pseudo-differential operator, and

$$
L^{(1)}=T L T^{-1}
$$

such that

$$
\partial_{t_{n}} L^{(1)}=\left[\left(L^{(1)}\right)_{\geq 1}^{n}, L^{(1)}\right]
$$

still holds for the transformed Lax operator $L^{(1)}$, then $T$ is called a gauge transformation operator of the mKP hierarchy. According to (3.2), one can easily obtain the lemma below.

Lemma 3.1. If the pseudo-differential operator $T$ satisfies

$$
\left(T L^{n} T^{-1}\right)_{\geq 1}=T\left(L^{n}\right)_{\geq 1} T^{-1}+T_{t_{n}} T^{-1},
$$

then $T$ is a gauge transformation operator of the $m K P$ hierarchy.

Before the construction of the gauge transformation, the following basic identities on the pseudo-differential operator are needed.

aPlease note that the $\mathrm{mK} P$ hierarchy that we consider is the Kupershmidt-Kiso version, which is different from from those in L. A. Dickey's work about mKP hierarchy [8]. The existence of $\tau_{1}$ and $\tau_{0}$ makes the mKP hierarchy in KupershmidtKiso version become a relatively separate system, just like the KP hierarchy. 
Lemma 3.2. For any pseudo-differential operator $A$ and arbitrary functions $f, f_{1}, f_{2}, g, g_{1}$ and $g_{2}$ , one has the following operator identities:

$$
\begin{aligned}
& \left(f^{-1} A f\right)_{\geq 1}=f^{-1} A_{\geq 1} f-f^{-1} A_{\geq 1}(f), \\
& \left(f_{x}^{-1} \partial A \partial^{-1} f_{x}\right)_{\geq 1}=f_{x}^{-1} \partial A_{\geq 1} \partial^{-1} f_{x}-f_{x}^{-1}\left(A_{\geq 1}(f)\right)_{x}, \\
& \left(\partial^{-1} g A g^{-1} \partial\right)_{\geq 1}=\partial^{-1} g A_{\geq 1} g^{-1} \partial-\partial^{-1} g^{-1} A_{\geq 1}^{*}(g) \partial, \\
& \left(A f \partial^{-1}\right)_{<0}=A_{\geq 0}(f) \partial^{-1}+A_{<0} f \partial^{-1}, \\
& \left(\partial^{-1} g A\right)_{<0}=\partial^{-1} A_{\geq 0}^{*}(g)+\partial^{-1} g A_{<0}, \\
& f_{1} \partial^{-1} g_{1} f_{2} \partial^{-1} g_{2}=f_{1} \int g_{1} f_{2} d x \cdot \partial^{-1} g_{2}-f_{1} \partial^{-1} \int g_{1} f_{2} d x \cdot g_{2} .
\end{aligned}
$$

Proof. (3.4), (3.5), (3.7) and (3.8) can be found in [26, 27]. (3.9) can be obtained by direct computation. Therefore, we only prove (3.6) here. By using (3.8)

$$
\begin{aligned}
\left(\partial^{-1} g A g^{-1} \partial\right)_{\geq 1} & =\left(\partial^{-1} g A_{\geq 1} g^{-1}\right)_{\geq 0} \partial \\
& =\partial^{-1} g A_{\geq 1} g^{-1} \partial-\left(\partial^{-1} g A_{\geq 1} g^{-1}\right)_{<0} \partial \\
& =\partial^{-1} g A_{\geq 1} g^{-1} \partial-\partial^{-1} g^{-1} A_{\geq 1}^{*}(g) \partial .
\end{aligned}
$$

After the preparation above, one can find the following proposition $[26,29,30]$ about the mKP hierarchy by using Lemma 3.1 and Lemma 3.2.

Proposition 3.1. There are three elementary gauge transformation operators for the $m K P$ hierarchy, that is,

$$
\begin{aligned}
& T_{1}(\Phi)=\Phi^{-1} \\
& T_{2}(\Phi)=\Phi_{x}^{-1} \partial \\
& T_{3}(\Psi)=\partial^{-1} \Psi
\end{aligned}
$$

where $\Phi \neq 0$ and $\Psi$ are the eigenfunction and the adjoint eigenfunction of the $m K P$ hierarchy (see (2.17)) respectively, and in particular $\Phi$ is not a constant in $T_{2}$.

Further, one can obtain the following proposition.

Proposition 3.2. Under the gauge transformation operator $T_{1}(\Phi), T_{2}(\Phi)$ and $T_{3}(\Psi)$, the objects in the $m K P$ hierarchy are transformed in the way shown in Table I.

Table I. Elementary gauge transformations $m K P \rightarrow m K P$

\begin{tabular}{lclccc}
\hline \hline$L_{m K P} \rightarrow L_{m K P}^{(1)}$ & $Z^{(1)}=$ & $\Phi_{1}^{(1)}=$ & $\Psi_{1}^{(1)}=$ & $\tau_{0}^{(1)}=$ & $\tau_{1}^{(1)}=$ \\
\hline$T_{1}=\Phi^{-1}$ & $\Phi^{-1} Z$ & $\Phi^{-1} \Phi_{1}$ & $\Phi \Psi_{1}$ & $\tau_{0}$ & $\Phi \tau_{1}$ \\
$T_{2}=\Phi_{x}^{-1} \partial$ & $\Phi_{x}^{-1} \partial Z \partial^{-1}$ & $\Phi_{x}^{-1} \Phi_{1 x}$ & $\Phi_{x} \int \Psi_{1} d x$ & $\tau_{1}$ & $\Phi_{x} \tau_{1}^{2} / \tau_{0}$ \\
$T_{3}=\partial^{-1} \Psi$ & $\partial^{-1} \Psi Z \partial$ & $\int \Psi \Phi_{1} d x$ & $\left(\Psi_{1} / \Psi\right)_{x}$ & $\Psi \tau_{0}^{2} / \tau_{1}$ & $\tau_{0}$ \\
\hline \hline
\end{tabular}

where $\Phi_{1}$ and $\Psi_{1}$ are the different eigenfunction and the adjoint eigenfunction of $L_{m K P}$ from the generating functions $\Phi$ and $\Psi$ of the gauge transformation operators. 
Proof. Here we only take $T_{2}$ as an example and the others can be derived in the similar way. The actions of $T_{2}$ on $Z, \Phi_{1}$ and $\Psi_{1}$ can be obtained by direct computation. Therefore we mainly focus on the actions on $\tau_{0}$ and $\tau_{1}$. Firstly, from

$$
\begin{aligned}
Z^{(1)} & =\Phi_{x}^{-1} \partial Z \partial^{-1}=\Phi_{x}^{-1} \partial\left(z_{0}+z_{1} \partial^{-1}+\cdots\right) \partial^{-1} \\
& =\Phi_{x}^{-1} z_{0}+\Phi_{x}^{-1}\left(z_{0 x}+z_{1}\right) \partial^{-1}+\cdots
\end{aligned}
$$

one can get

$$
z_{0}^{(1)}=\Phi_{x}^{-1} z_{0}, \quad z_{1}^{(1)}=\Phi_{x}^{-1}\left(z_{0 x}+z_{1}\right)
$$

Then by (2.16), one can find

$$
-\partial_{x} \ln \tau_{0}^{(1)}=\frac{z_{1}^{(1)}}{z_{0}^{(1)}}=\partial_{x} \ln z_{0}+\frac{z_{1}}{z_{0}}=\partial_{x} \ln \frac{z_{0}}{\tau_{0}}
$$

Therefore according to (2.16) and (3.14),

$$
\tau_{0}^{(1)}=\frac{\tau_{0}}{z_{0}}=\tau_{1}, \quad \tau_{1}^{(1)}=\frac{\tau_{0}^{(1)}}{z_{0}^{(1)}}=\frac{\Phi_{x} \tau_{1}^{2}}{\tau_{0}} .
$$

Remark: Note that in $[29,30]$, there is another gauge transformation operator $T=\partial^{-1} \int \Psi d x \cdot \partial$, which can be viewed as the composition of $T_{2}$ and $T_{3}$, that is, $T_{3}\left(\Psi^{(1)}\right) T_{2}(\Phi)=\partial^{-1} \Psi^{(1)} \Phi \partial=$ $\partial^{-1} \int \Psi d x \cdot \partial$, with $\Psi^{(1)}=T_{2}(\Phi)(\Psi)$.

Denote the successive applications of gauge transformation operators $T_{i}$ with $i=1,2,3$ as

$$
\begin{aligned}
& T_{1}^{(n)}\left(\Phi_{1}, \cdots, \Phi_{n}\right) \triangleq T_{1}\left(\Phi_{n}^{(n-1)}\right) \cdots T_{1}\left(\Phi_{3}^{(2)}\right) T_{1}\left(\Phi_{2}^{(1)}\right) T_{1}\left(\Phi_{1}\right) \\
& T_{2}^{(n)}\left(\Phi_{1}, \cdots, \Phi_{n}\right) \triangleq T_{2}\left(\Phi_{n}^{(n-1)}\right) \cdots T_{2}\left(\Phi_{3}^{(2)}\right) T_{2}\left(\Phi_{2}^{(1)}\right) T_{2}\left(\Phi_{1}\right) \\
& T_{3}^{(n)}\left(\Psi_{1}, \cdots, \Psi_{n}\right) \triangleq T_{3}\left(\Psi_{n}^{(n-1)}\right) \cdots T_{3}\left(\Psi_{3}^{(2)}\right) T_{3}\left(\Psi_{2}^{(1)}\right) T_{3}\left(\Psi_{1}\right) .
\end{aligned}
$$

Here $\Phi_{i}$ and $\Psi_{i}$ are the eigenfunctions and the adjoint eigenfunctions of the mKP hierarchy which are different from each other, $\Phi_{k}^{(m)}=T_{i}^{(m)}\left(\Phi_{1}, \cdots, \Phi_{m}\right)\left(\Phi_{k}\right)$ and $\Psi_{k}^{(m)}=T_{i}^{(m)}\left(\Psi_{1}, \cdots, \Psi_{m}\right)\left(\Psi_{k}\right)$. The results of the successive applications of gauge transformation operators $T_{i}$ with $i=1,2,3$ are listed in the proposition below. 


\section{Proposition 3.3.}

(1) $T_{1}^{(n)}\left(\Phi_{1}, \cdots, \Phi_{n}\right)=\Phi_{n}^{-1}$,

(2) $T_{2}^{(n)}\left(\Phi_{1}, \cdots, \Phi_{n}\right)=\frac{1}{W_{n+1}\left(1, \Phi_{1}, \cdots, \Phi_{n}\right)}\left|\begin{array}{cccc}\Phi_{1 x} & \cdots & \Phi_{n-1, x} & \partial \\ \vdots & \vdots & \vdots & \vdots \\ \Phi_{1}^{(i)} & \cdots & \Phi_{n-1}^{(i)} & \partial^{i} \\ \vdots & \vdots & \vdots & \vdots \\ \Phi_{1}^{(n)} & \cdots & \Phi_{n-1}^{(n)} & \partial^{n}\end{array}\right|$,

(3) $T_{3}^{(n)}\left(\Psi_{1}, \cdots, \Psi_{n}\right)=\frac{(-1)^{n+1}}{W_{n-1}\left(\Psi_{1}, \cdots, \Psi_{n-1}\right)}\left|\begin{array}{ccccc}\Psi_{1} & \Psi_{1}^{(1)} & \cdots & \Psi_{1}^{(n-2)} & \partial^{-1} \Psi_{1} \\ \vdots & \vdots & \vdots & \vdots & \vdots \\ \Psi_{n} \Psi_{n}^{(1)} & \cdots & \Psi_{n}^{(n-2)} & \partial^{-1} \Psi_{n}\end{array}\right|$,

where $W_{n} \equiv W_{n}\left(\Phi_{1}, \Phi_{2}, \cdots, \Phi_{n}\right)$ is the Wronskian determinant of $\Phi_{1}, \Phi_{2}, \cdots, \Phi_{n}$. And the determinant of $T_{3}^{(n)}$ is expanded by the last column and the functions are on the left-hand side.

Proof. (1)

$$
\begin{aligned}
T_{1}^{(n)} & =T_{1}\left(\Phi_{n}^{(n-1)}\right) \cdots T_{1}\left(\Phi_{3}^{(2)}\right) T_{1}\left(\Phi_{2}^{(1)}\right) T_{1}\left(\Phi_{1}\right) \\
& =\left(\Phi_{1} \Phi_{2}^{(1)} \cdots \Phi_{n-2}^{(n-3)} \Phi_{n-1}^{(n-2)} \Phi_{n}^{(n-1)}\right)^{-1} \\
& =\left(\Phi_{1} \Phi_{2}^{(1)} \cdots \Phi_{n-2}^{(n-3)} \Phi_{n}^{(n-2)}\right)^{-1} \\
& =\left(\Phi_{1} \Phi_{2}^{(1)} \cdots \Phi_{n}^{(n-3)}\right)^{-1}=\cdots=\Phi_{n}^{-1}
\end{aligned}
$$

where we have used the following fact $\Phi_{k}^{(k-1)} \Phi_{j}^{(k)}=\Phi_{j}^{(k-1)}$, with $\Phi_{j}^{(k)}=T_{1}\left(\Phi_{k}^{(k-1)}\right)\left(\Phi_{j}^{(k-1)}\right)$.

(2) Assume $T_{2}^{(n)}=\sum_{i=1}^{n} a_{i} \partial^{i}$. Since $T_{2}^{(n)}\left(\Phi_{i}\right)=0, i=1,2, \cdots, n-1$ and $T_{2}^{(n)}\left(\Phi_{n}\right)=1$, one can obtain the following linear equation group,

$$
\left\{\begin{array}{l}
\sum_{i=1}^{n} a_{i} \Phi_{k}^{(i)}=0, \quad k=1,2, \cdots n-1 \\
\sum_{i=1}^{n} a_{i} \Phi_{n}^{(i)}=1
\end{array}\right.
$$

Thus

$$
a_{i}=(-1)^{i+n} \frac{1}{W_{n+1}\left(1, \Phi_{1}, \cdots, \Phi_{n}\right)}\left|\begin{array}{ccc}
\Phi_{1 x} & \cdots & \Phi_{n-1, x} \\
\vdots & \vdots & \vdots \\
\Phi_{1}^{(i-1)} & \cdots & \Phi_{n-1}^{(i-1)} \\
\Phi_{1}^{(i+1)} & \cdots & \Phi_{n-1}^{(i+1)} \\
\vdots & \vdots & \vdots \\
\Phi_{1}^{(n)} & \cdots & \Phi_{n-1}^{(n)}
\end{array}\right| .
$$

The substitution of (3.25) for $T_{2}^{(n)}=\sum_{i=1}^{n} a_{i} \partial^{i}$ leads to (3.21).

(3) Firstly, we can prove that $T_{3}^{(n)}$ has the form of $T_{3}^{(n)}=\sum_{i=1}^{n} b_{i} \partial^{-1} \Psi_{i}$ with $b_{n}=(-1)^{n+1}$ by 
induction. In fact, we can assume $T_{3}^{(n-1)}\left(\Psi_{2}^{(1)}, \cdots, \Psi_{n}^{(1)}\right)=\sum_{i=2}^{n} b_{i} \partial^{-1} \Psi_{i}^{(1)}$ with $b_{n}=(-1)^{n}$. Then according to (3.9) and Proposition 3.2, one can find

$$
\begin{aligned}
T_{3}^{(n)} & =T_{3}^{(n-1)}\left(\Psi_{2}^{(1)}, \cdots, \Psi_{n}^{(1)}\right) T_{3}\left(\Psi_{1}\right)=\sum_{i=2}^{n} b_{i} \partial^{-1} \Psi_{i}^{(1)} \partial^{-1} \Psi_{1} \\
& =\sum_{i=2}^{n}\left(b_{i} \int \Psi_{i}^{(1)} d x \cdot \partial^{-1} \Psi_{1}-b_{i} \partial^{-1} \int \Psi_{i}^{(1)} d x \cdot \Psi_{1}\right) \\
& =\sum_{i=2}^{n}\left(b_{i} \int \Psi_{i}^{(1)} d x \cdot \partial^{-1} \Psi_{1}-b_{i} \partial^{-1} \Psi_{i}\right)
\end{aligned}
$$

still holds with the term $(-1)^{n+1} \partial^{-1} \Psi_{n}$.

Then by comparing the fact $T_{3}^{(n)}=\alpha_{-n} \partial^{-n}+$ (lower order terms) with the form of $T_{3}^{(n)}$ given above, one can obtain the following linear equation group,

$$
\left\{\begin{array}{l}
\sum_{i=1}^{n} b_{i} \Psi_{i}^{(k)}=0, \quad k=0,1,2, \cdots n-2, \\
b_{n}=(-1)^{n+1}
\end{array}\right.
$$

Therefore

$$
b_{i}=\frac{(-1)^{i+1}}{W_{n-1}\left(\Psi_{1}, \cdots, \Psi_{n-1}\right)}\left|\begin{array}{cccccc}
\Psi_{1} & \ldots & \Psi_{i-1} & \Psi_{i+1} & \ldots & \Psi_{n} \\
\Psi_{1 x} & \ldots & \Psi_{i-1, x} & \Psi_{i+1, x} & \ldots & \Psi_{n, x} \\
\vdots & \vdots & \vdots & \vdots & \vdots & \vdots \\
\Psi_{1}^{(n-2)} & \ldots & \Psi_{i-1}^{(n-2)} & \Psi_{i+1}^{(n-2)} & \ldots & \Psi_{n}^{(n-2)}
\end{array}\right|,
$$

which leads to (3.22).

Assume $L$ be the Lax operator of the mKP hierarchy, $\Phi_{1}$ and $\Phi_{2}$ be two nonzero independent eigenfunctions, and $\Psi_{1}$ and $\Psi_{2}$ be two independent adjoint eigenfunctions. we consider the following diagram

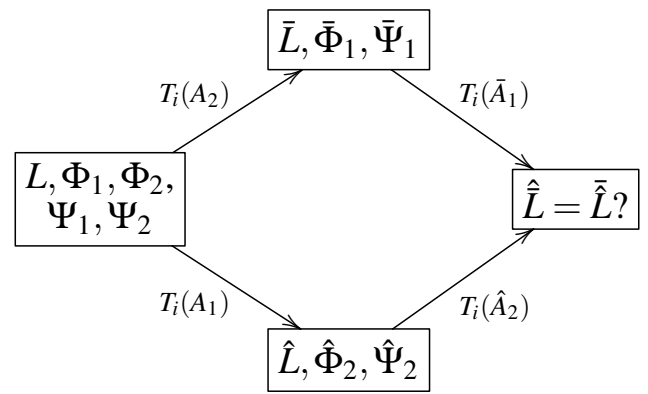

Figure 1. The Bianchi diagram for $T_{i}, i=1,2,3$.

where $i=1,2,3$ and

$$
\begin{aligned}
& A_{i}=\left\{\begin{array}{ll}
\Phi, & i=1,2 \\
\Psi, & i=3
\end{array},\right. \\
& \hat{L}=T_{i}\left(A_{1}\right) L T_{i}\left(A_{1}\right)^{-1}, \quad \hat{\Phi}_{2}=T_{i}\left(A_{1}\right)\left(\Phi_{2}\right), \quad \hat{\Psi}_{2}=\left(T_{i}^{*}\left(A_{1}\right)\right)^{-1}\left(\Psi_{2}\right), \\
& \bar{L}=T_{i}\left(A_{2}\right) L T_{i}\left(A_{2}\right)^{-1}, \quad \bar{\Phi}_{1}=T_{i}\left(A_{2}\right)\left(\Phi_{1}\right), \quad \bar{\Psi}_{1}=\left(T_{i}^{*}\left(A_{2}\right)\right)^{-1}\left(\Psi_{1}\right), \\
& \hat{\bar{L}}=T_{i}\left(\bar{A}_{1}\right) \bar{L} T_{i}\left(\bar{A}_{1}\right)^{-1}, \quad \overline{\hat{L}}=T_{i}\left(\hat{A}_{2}\right) \hat{L} T_{i}\left(\hat{A}_{2}\right)^{-1} .
\end{aligned}
$$


The question is whether this diagram will commute, i.e.,

$$
\begin{aligned}
& T_{i}^{(2)}\left(\Phi_{1}, \Phi_{2}\right)=T_{i}^{(2)}\left(\Phi_{2}, \Phi_{1}\right), \quad i=1,2, \\
& T_{3}^{(2)}\left(\Psi_{1}, \Psi_{2}\right)=T_{3}^{(2)}\left(\Psi_{2}, \Psi_{1}\right) .
\end{aligned}
$$

In fact, according to Proposition 3.3,

$$
\begin{aligned}
& T_{1}^{(2)}\left(\Phi_{1}, \Phi_{2}\right)-T_{1}^{(2)}\left(\Phi_{2}, \Phi_{1}\right)=\Phi_{2}^{-1}-\Phi_{1}^{-1} \neq 0 \\
& T_{2}^{(2)}\left(\Phi_{1}, \Phi_{2}\right)-T_{2}^{(2)}\left(\Phi_{2}, \Phi_{1}\right)=\frac{1}{W_{3}\left(1, \Phi_{1}, \Phi_{2}\right)}\left|\begin{array}{cc}
\Phi_{1 x}+\Phi_{2 x} & \partial \\
\Phi_{1 x x}+\Phi_{2 x x} & \partial^{2}
\end{array}\right| \neq 0, \\
& T_{3}^{(2)}\left(\Psi_{1}, \Psi_{2}\right)-T_{3}^{(2)}\left(\Psi_{2}, \Psi_{1}\right)=\left(\Psi_{2} / \Psi_{1}+1\right) \partial^{-1} \Psi_{1}-\left(\Psi_{1} / \Psi_{2}+1\right) \partial^{-1} \Psi_{2} \neq 0 .
\end{aligned}
$$

Therefore, the Bianchi diagrams for $T_{i}, i=1,2,3$ does not commute. Further, according to Proposition 3.2, one can also find that

$$
\begin{aligned}
T_{1}\left(\Phi_{2}^{(1)}\right) T_{2}\left(\Phi_{1}\right) & \neq T_{2}\left(\Phi_{1}^{(1)}\right) T_{1}\left(\Phi_{2}\right) \\
T_{1}\left(\Phi^{(1)}\right) T_{3}(\Psi) & \neq T_{3}\left(\Psi^{(1)}\right) T_{1}(\Phi) \\
T_{2}\left(\Phi^{(1)}\right) T_{3}(\Psi) & \neq T_{3}\left(\Psi^{(1)}\right) T_{2}(\Phi) .
\end{aligned}
$$

So $T_{i}$ with $i=1,2,3$ can not commute with each other, that is,

$$
T_{i} T_{j} \neq T_{j} T_{i}, \quad i, j=1,2,3 .
$$

This fact tells us that the gauge transformation operators $T_{i}$ with $i=1,2,3$ are not convenient when solving the mKP hierarchy. Thus we must seek the other kinds of gauge transformation operators which can commute with each other in the mKP hierarchy, that is, $T_{D}(\Phi)$ and $T_{I}(\Psi)$.

\section{Gauge Transformation operators $T_{D}(\Phi)$ and $T_{I}(\Psi)$}

Since 1 is the eigenfunction of the mKP hierarchy (see (2.17)), one can define

$$
\begin{aligned}
& T_{D}(\Phi) \triangleq T_{2}\left(1^{(1)}\right) T_{1}(\Phi)=\left(\Phi^{-1}\right)_{x}^{-1} \partial \Phi^{-1} \\
& T_{I}(\Psi) \triangleq T_{1}\left(1^{(1)}\right) T_{3}(\Psi)=\left(\int \Psi d x\right)^{-1} \partial^{-1} \Psi .
\end{aligned}
$$

Here $T_{D}$ is introduced in [26], while as far as I know $T_{I}$ is presented for the first time in the literature. Then by the direct computation similar to Proposition 3.2, one can obtain the following proposition.

Proposition 4.1. Under the gauge transformation operator $T_{D}(\Phi)$ and $T_{I}(\Psi)$, the objects in the $m K P$ hierarchy are transformed in the way shown in Table II.

\section{Table II. gauge transformations $T_{D}(\Phi)$ and $T_{I}(\Psi)$}

\begin{tabular}{cccccc}
\hline \hline$L_{m K P} \rightarrow L_{m K P}^{(1)}$ & $Z^{(1)}=$ & $\Phi_{1}^{(1)}=$ & $\Psi_{1}^{(1)}=$ & $\tau_{0}^{(1)}=$ & $\tau_{1}^{(1)}=$ \\
\hline$T_{D}(\Phi)$ & $T_{D}(\Phi) Z \partial^{-1}$ & $\left(\Phi_{1} / \Phi\right)_{x} /\left(\Phi^{-1}\right)_{x}$ & $\left(\Phi^{-1}\right)_{x} \cdot \int \Phi \Psi_{1} d x$ & $\Phi \tau_{1}$ & $-\Phi_{x} \tau_{1}^{2} / \tau_{0}$ \\
$T_{I}(\Psi)$ & $T_{I}(\Psi) Z \partial$ & $\int \Psi \Phi_{1} d x / \int \Psi d x$ & $\left(\Psi_{1} / \Psi\right)_{x} \cdot \int \Psi d x$ & $\Psi \tau_{0}^{2} / \tau_{1}$ & $\tau_{0} \cdot \int \Psi d x$ \\
\hline \hline
\end{tabular}


According to Table II, one finds that

$$
\begin{aligned}
T_{D}(\Phi)(\Phi) & =0, \quad\left(T_{I}(\Psi)^{-1}\right)^{*}(\Psi)=0, \\
T_{D}(\Phi)(1) & =1, T_{I}(\Psi)(1)=1 .
\end{aligned}
$$

It can be proved that $T_{D}(\Phi)$ and $T_{I}(\Psi)$ are commute with each other, i.e.,

$$
\begin{aligned}
T_{D}\left(\Phi_{2}^{(1)}\right) T_{D}\left(\Phi_{1}^{(0)}\right) & =T_{D}\left(\Phi_{1}^{(1)}\right) T_{D}\left(\Phi_{2}^{(0)}\right), \\
T_{D}\left(\Phi^{(1)}\right) T_{I}\left(\Psi^{(0)}\right) & =T_{I}\left(\Psi^{(1)}\right) T_{D}\left(\Phi^{(0)}\right), \\
T_{I}\left(\Psi_{2}^{(1)}\right) T_{I}\left(\Psi_{1}^{(0)}\right) & =T_{I}\left(\Psi_{1}^{(1)}\right) T_{I}\left(\Psi_{2}^{(0)}\right) .
\end{aligned}
$$

Therefore, $T_{D}(\Phi)$ and $T_{I}(\Psi)$ are more applicable in the case of the mKP hierarchy.

Consider the following chain of the gauge transformation operators $T_{D}(\Phi)$ and $T_{I}(\Psi)$

$$
\begin{aligned}
& L \stackrel{T_{D}\left(\Phi_{1}\right)}{\longrightarrow} L^{(1)} \stackrel{T_{D}\left(\Phi_{2}^{(1)}\right)}{\longrightarrow} L^{(2)} \rightarrow \cdots \rightarrow L^{(n-1)} \stackrel{T_{D}\left(\Phi_{n}^{(n-1)}\right)}{\longrightarrow} L^{(n)} \\
& \stackrel{T_{I}\left(\Psi_{1}^{(n)}\right)}{\longrightarrow} L^{(n+1)} \stackrel{T_{I}\left(\Psi_{1}^{(n+1)}\right)}{\longrightarrow} \cdots \rightarrow L^{(n+k-1)} \stackrel{T_{I}\left(\Psi_{k}^{(n+k-1)}\right)}{\longrightarrow} L^{(n+k)} .
\end{aligned}
$$

Denote

$$
T^{(n, k)}=T_{I}\left(\Psi_{k}^{(n+k-1)}\right) \cdots T_{I}\left(\Psi_{1}^{(n)}\right) T_{D}\left(\Phi_{n}^{(n-1)}\right) \cdots T_{D}\left(\Phi_{2}^{(1)}\right) T_{D}\left(\Phi_{1}\right) .
$$

Next, we will compute the explicit form of $T^{(n, k)}$ in terms of $\Phi_{i}$ and $\Psi_{i}$. Before this, the lemma below is needed.

Lemma 4.1. $T^{(0, k)}$ and $T^{(n, 0)}$ have the following form

$$
\begin{aligned}
T^{(0, k)} & =\sum_{i=1}^{k} \alpha_{i} \partial^{-1} \Psi_{i}, \\
\left(T^{(n, 0)}\right)^{-1} & =\sum_{i=1}^{n} \Phi_{i} \partial^{-1} \beta_{i} .
\end{aligned}
$$

Proof. This lemma can be proved by induction. Here we only prove the first identity, since the second one is almost the same. Assume this lemma holds for $k-1$, then according to (3.9) and Table II

$$
\begin{aligned}
T^{(0, k)}= & \sum_{i=2}^{k} \alpha_{i} \partial^{-1} \Psi_{i}^{(1)} \cdot\left(\int \Psi_{1} d x\right)^{-1} \cdot \partial^{-1} \Psi_{1} \\
= & \sum_{i=2}^{k} \alpha_{i} \cdot \int\left(\Psi_{i}^{(1)}\left(\int \Psi_{1} d x\right)^{-1}\right) d x \cdot \partial^{-1} \Psi_{1} \\
& -\sum_{i=2}^{k} \alpha_{i} \partial^{-1} \cdot \int\left(\Psi_{i}^{(1)}\left(\int \Psi_{1} d x\right)^{-1}\right) d x \cdot \Psi_{1} \\
= & \sum_{i=2}^{k}\left(\alpha_{i} \Psi_{i} / \Psi_{1} \partial^{-1} \Psi_{1}-\alpha_{i} \partial^{-1} \Psi_{i}\right),
\end{aligned}
$$

by noting that

$$
\int\left(\Psi_{i}^{(1)}\left(\int \Psi_{1} d x\right)^{-1}\right) d x \cdot \Psi_{1}=\Psi_{i}
$$


The generalized Wronskian determinant [9] is needed in the next propositions, which is defined in the following form

$$
\begin{aligned}
I W_{k, n} & \equiv I W_{k, n}\left(\Psi_{k}, \Psi_{k-1}, \cdots, \Psi_{1} ; \Phi_{1}, \cdots, \Phi_{n}\right) \\
& =\left|\begin{array}{cccc}
\int \Phi_{1} \Psi_{k} d x & \int \Phi_{2} \Psi_{k} d x & \cdots & \int \Phi_{n} \Psi_{k} d x \\
\int \Phi_{1} \Psi_{k-1} d x & \int \Phi_{2} \Psi_{k-1} d x & \cdots & \int \Phi_{n} \Psi_{k-1} d x \\
\vdots & \vdots & \vdots & \vdots \\
\int \Phi_{1} \Psi_{1} d x & \int \Phi_{2} \Psi_{1} d x & \cdots & \int \Phi_{n} \Psi_{1} d x \\
\Phi_{1} & \Phi_{2} & \cdots & \Phi_{n} \\
\Phi_{1 x} & \Phi_{2 x} & \cdots & \Phi_{n x} \\
\vdots & \vdots & \vdots & \vdots \\
\Phi_{1}^{(n-k-1)} & \Phi_{2}^{(n-k-1)} & \cdots & \Phi_{n}^{(n-k-1)}
\end{array}\right| .
\end{aligned}
$$

In particular,

$$
I W_{0, n}=W_{n}\left(\Phi_{1}, \Phi_{2}, \cdots, \Phi_{n}\right),
$$

which is Wronskian determinant of $\Phi_{1}, \Phi_{2}, \cdots, \Phi_{n}$.

Proposition 4.2. When $n>k, T^{(n, k)}$ and $\left(T^{(n, k)}\right)^{-1}$ have the following forms

$$
\begin{aligned}
& T^{(n, k)}=\frac{1}{I W_{k, n+1}\left(\Psi_{k}, \Psi_{k-1}, \cdots, \Psi_{1} ; \Phi_{1}, \cdots, \Phi_{n}, 1\right)} \\
& \left|\begin{array}{ccccc}
\int \Phi_{1} \Psi_{k} d x & \int \Phi_{2} \Psi_{k} d x & \cdots & \int \Phi_{n} \Psi_{k} d x & \partial^{-1} \Psi_{k} \\
\int \Phi_{1} \Psi_{k-1} d x & \int \Phi_{2} \Psi_{k-1} d x & \cdots & \int \Phi_{n} \Psi_{k-1} d x & \partial^{-1} \Psi_{k-1} \\
\vdots & \vdots & \vdots & \vdots & \vdots \\
\int \Phi_{1} \Psi_{1} d x & \int \Phi_{2} \Psi_{1} d x & \cdots & \int \Phi_{n} \Psi_{1} d x & \partial^{-1} \Psi_{1} \\
\Phi_{1} & \Phi_{2} & \cdots & \Phi_{n} & 1 \\
\Phi_{1 x} & \Phi_{2 x} & \cdots & \Phi_{n x} & \partial \\
\vdots & \vdots & \vdots & \vdots & \vdots \\
\Phi_{1}^{(n-k)} & \Phi_{2}^{(n-k)} & \cdots & \Phi_{n}^{(n-k)} & \partial^{n-k}
\end{array}\right|
\end{aligned}
$$

and

$$
\begin{aligned}
& \left(T^{(n, k)}\right)^{-1}=(-1)^{n-1} \frac{I W_{k, n+1}\left(\Psi_{k}, \Psi_{k-1}, \cdots, \Psi_{1} ; \Phi_{1}, \cdots, \Phi_{n}, 1\right)}{I W_{k, n}\left(\Psi_{k}, \Psi_{k-1}, \cdots, \Psi_{1} ; \Phi_{1}, \cdots, \Phi_{n}\right)^{2}}
\end{aligned}
$$

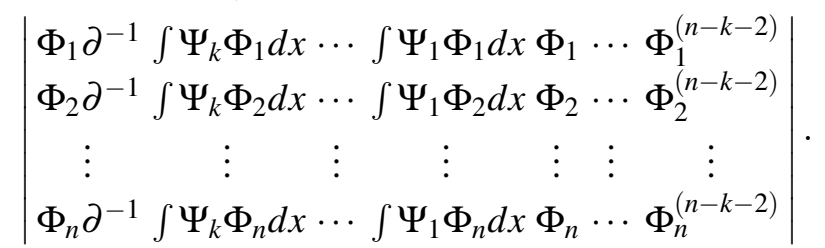

Here the determinant of $T^{(n, k)}$ is expanded by the last column and the functions are on the left-hand side. And when computing $\left(T^{(n, k)}\right)^{-1}$, the determinant of $\left(T^{(n, k)}\right)^{-1}$ is expanded by the first column and the functions are on the right-hand side, and also the coefficient function before the determinant should be placed after the operators $\Phi_{i} \partial^{-1}$. 
Proof. When $n>k$, according to (4.8) and the commutativity of $T_{D}$ and $T_{I}$ given in (4.5)-(4.7), one can rewrite $T^{(n, k)}$ as

$$
T^{(n, k)}=A T^{(0, k)}=B^{-1} T^{(n, 0)},
$$

where

$$
\begin{aligned}
& A=T_{D}\left(\Phi_{n}^{(n+k-1)}\right) \cdots T_{D}\left(\Phi_{2}^{(k+1)}\right) T_{D}\left(\Phi_{1}^{(k)}\right) \\
& B=\left(T_{I}\left(\Psi_{k}^{(n+k-1)}\right) T_{I}\left(\Psi_{k-1}^{(n+k-2)}\right) \cdots T_{I}\left(\Psi_{1}^{(n)}\right)\right)^{-1}
\end{aligned}
$$

Then by using Lemma 3.2 and Lemma 4.1, and also the fact $\left(T^{(n, k)}\right)^{-1}=\left(\left(T^{(n, k)}\right)^{-1}\right)_{-}$,

$$
\begin{gathered}
\left(T^{(n, k)}\right)_{-}=\left(A T^{(0, k)}\right)_{-}=\left(A \sum_{i=1}^{k} \alpha_{i} \partial^{-1} \Psi_{i}\right)_{-}=\sum_{i=1}^{k} A\left(\alpha_{i}\right) \partial^{-1} \Psi_{i} \\
\left(T^{(n, k)}\right)^{-1}=\left(\left(T^{(n, 0)}\right)^{-1} B\right)_{-}=\left(\sum_{i=1}^{n} \Phi_{i} \partial^{-1} \beta_{i} B\right)_{-}=\sum_{i=1}^{n} \Phi_{i} \partial^{-1} B^{*}\left(\beta_{i}\right)
\end{gathered}
$$

Thus $T^{(n, k)}$ and $\left(T^{(n, k)}\right)^{-1}$ have the following forms

$$
\begin{aligned}
& T^{(n, k)}=\sum_{i=0}^{n-k} a_{i} \partial^{i}+\sum_{i=-k}^{-1} a_{i} \partial^{-1} \Psi_{-i} \\
& \left(T^{(n, k)}\right)^{-1}=\sum_{j=1}^{n} \Phi_{j} \partial^{-1} b_{j}
\end{aligned}
$$

where $a_{i}$ and $b_{j}$ are the functions that will be determined below.

Then from Table II, one can find

$$
T^{(n, k)}\left(\Phi_{i}\right)=0, T^{(n, k)}(1)=1, i=1,2, \cdots, n,
$$

that is

$$
\left\{\begin{array}{l}
\sum_{i=0}^{n-k} a_{i} \Phi_{j}^{(i)}+\sum_{i=-k}^{-1} a_{i} \int \Psi_{-i} \Phi_{j} d x=0, j=1,2, \cdots, n, \\
a_{0}+\sum_{i=-k}^{-1} a_{i} \int \Psi_{-i} d x=1
\end{array}\right.
$$

where (4.21) is used. By solving this equation, (4.14) can be proved.

On the other hand, (4.21), (4.22) and Table II can lead to

$$
\begin{aligned}
& \left(\left(T^{(n, k)}\right)^{-1}\right)^{*}=\sum_{l=0}^{+\infty}(-1)^{l+1} \sum_{j=1}^{n} b_{j} \Phi_{j}^{(l)} \partial^{-1-l} \\
& \left(\left(T^{(n, k)}\right)^{-1}\right)^{*}=(-1)^{n-k} a_{n-k}^{-1} \partial^{-n+k}+(\text { lower order terms }) \\
& \left(\left(T^{(n, k)}\right)^{-1}\right)^{*}\left(\Psi_{j}\right)=0
\end{aligned}
$$


Thus

$$
\left\{\begin{array}{l}
\sum_{i=1}^{n} b_{i} \int \Phi_{i} \Psi_{j} d x=0, j=k, k-1, \cdots, 1, \\
\sum_{i=1}^{n} b_{i} \Phi_{i}^{(j)}=0, j=0,1,2, \cdots, n-k-2, \\
\sum_{i=1}^{n} b_{i} \Phi_{i}^{(n-k-1)}=a_{n-k}^{-1}=\frac{I W_{k, n+1}\left(\Psi_{k}, \Psi_{k-1}, \cdots, \Psi_{1} ; \Phi_{1}, \cdots, \Phi_{n}, 1\right)}{I W_{k, n}\left(\Psi_{k}, \Psi_{k-1}, \cdots, \Psi_{1} ; \Phi_{1}, \cdots, \Phi_{n}\right)},
\end{array}\right.
$$

which gives rise to (4.15).

By the same manner as the case $n>k$, one can show that $T^{(n, n)}$ and $\left(T^{(n, n)}\right)^{-1}$ have the similar forms

$$
\begin{aligned}
& T^{(n, n)}=a_{0}+\sum_{i=-n}^{-1} a_{i} \partial^{-1} \Psi_{-i}, \\
& \left(T^{(n, n)}\right)^{-1}=a_{0}^{-1}+\sum_{j=1}^{n} \Phi_{j} \partial^{-1} b_{j},
\end{aligned}
$$

and thus have the following proposition.

Proposition 4.3. When $n=k, T^{(n, k)}$ and $\left(T^{(n, k)}\right)^{-1}$ have the following forms

$$
\begin{aligned}
T^{(n, n)}= & \frac{1}{I W_{n, n+1}\left(\Psi_{n}, \Psi_{n-1}, \cdots, \Psi_{1} ; \Phi_{1}, \cdots, \Phi_{n}, 1\right)} \\
& \left|\begin{array}{ccccc}
\int \Phi_{1} \Psi_{n} d x & \int \Phi_{2} \Psi_{n} d x & \cdots & \int \Phi_{n} \Psi_{n} d x & \partial^{-1} \Psi_{n} \\
\int \Phi_{1} \Psi_{n-1} d x & \int \Phi_{2} \Psi_{n-1} d x & \cdots & \int \Phi_{n} \Psi_{n-1} d x & \partial^{-1} \Psi_{n-1} \\
\vdots & \vdots & \vdots & \vdots & \vdots \\
\int \Phi_{1} \Psi_{1} d x & \int \Phi_{2} \Psi_{1} d x & \cdots & \int \Phi_{n} \Psi_{1} d x & \partial^{-1} \Psi_{1} \\
\Phi_{1} & \Phi_{2} & \cdots & \Phi_{n} & 1
\end{array}\right|,
\end{aligned}
$$

and

$$
\begin{aligned}
& \left(T^{(n, n)}\right)^{-1}=-\frac{I W_{n, n+1}\left(\Psi_{n}, \Psi_{n-1}, \cdots, \Psi_{1} ; \Phi_{1}, \cdots, \Phi_{n}, 1\right)}{I W_{n, n}\left(\Psi_{n}, \Psi_{n-1}, \cdots, \Psi_{1} ; \Phi_{1}, \cdots, \Phi_{n}\right)^{2}} \\
& \left|\begin{array}{ccccc}
-1 & \Psi_{n} & \Psi_{n-1} & \cdots & \Psi_{1} \\
\Phi_{1} \partial^{-1} & \int \Psi_{n} \Phi_{1} d x & \int \Psi_{n-1} \Phi_{1} d x & \cdots & \int \Psi_{1} \Phi_{1} d x \\
\Phi_{2} \partial^{-1} & \int \Psi_{n} \Phi_{2} d x & \int \Psi_{n-1} \Phi_{2} d x & \cdots & \int \Psi_{1} \Phi_{2} d x \\
\vdots & \vdots & \vdots & \vdots & \vdots \\
\Phi_{n} \partial^{-1} & \int \Psi_{n} \Phi_{n} d x & \int \Psi_{n-1} \Phi_{n} d x & \cdots & \int \Psi_{1} \Phi_{n} d x
\end{array}\right|
\end{aligned}
$$

Here the determinant of $T^{(n, k)}$ is expanded by the last column and the functions are on the left-hand side. And when computing $\left(T^{(n, k)}\right)^{-1}$, the determinant of $\left(T^{(n, k)}\right)^{-1}$ is expanded by the first column and the functions are on the right-hand side, and also the coefficient function before the determinant should be placed after the operators $\Phi_{i} \partial^{-1}$.

As for the case $n<k$, one has the proposition below. 
Proposition 4.4. When $n<k, T^{(n, k)}$ and $\left(T^{(n, k)}\right)^{-1}$ have the following forms

$$
\begin{aligned}
& T^{(n, k)}=\frac{(-1)^{k-1}}{I W_{n+1, k}\left(1, \Phi_{n}, \Phi_{n-1}, \cdots, \Phi_{1} ; \Psi_{1}, \cdots, \Psi_{k}\right)}
\end{aligned}
$$

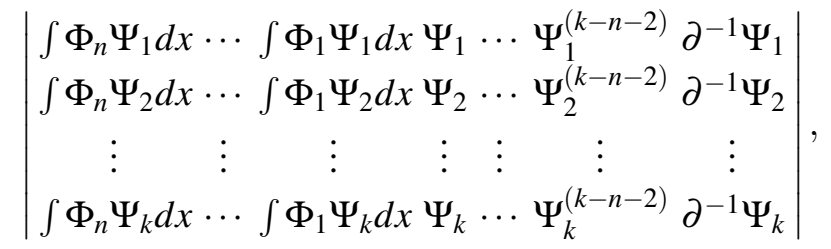

and

$$
\begin{aligned}
&\left(T^{(n, k)}\right)^{-1}= \frac{I W_{n+1, k}\left(1, \Phi_{n}, \Phi_{n-1}, \cdots, \Phi_{1} ; \Psi_{1}, \cdots, \Psi_{k}\right)}{I W_{n, k}\left(\Phi_{n}, \Phi_{n-1}, \cdots, \Phi_{1} ; \Psi_{1}, \cdots, \Psi_{k}\right)^{2}} \\
&\left|\begin{array}{cccc}
-\Phi_{n} \partial^{-1} & \int \Phi_{n} \Psi_{1} d x & \cdots & \Phi_{n} \Psi_{k} d x \\
\vdots & \vdots & \vdots & \vdots \\
-\Phi_{1} \partial^{-1} & \int \Phi_{1} \Psi_{1} d x & \cdots & \int \\
1 & \Psi_{1} \Psi_{k} d x \\
-\partial & \Psi_{1 x} & \cdots & \Psi_{k} \\
\vdots & \vdots & \vdots & \vdots \\
(-\partial)^{k-n} & \Psi_{1}^{(k-n)} & \ldots & \Psi_{k}^{(k-n)}
\end{array}\right|
\end{aligned}
$$

Here the determinant of $T^{(n, k)}$ is expanded by the last column and the functions are on the left-hand side. And when computing $\left(T^{(n, k)}\right)^{-1}$, the determinant of $\left(T^{(n, k)}\right)^{-1}$ is expanded by the first column and the functions are on the right-hand side, and also the coefficient function before the determinant should be placed after the operators $\Phi_{i} \partial^{-1}$ and $\partial^{i}$.

Proof. Firstly when $n<k, T^{(n, k)}$ and $\left(T^{(n, k)}\right)^{-1}$ have the following forms

$$
\begin{aligned}
& T^{(n, k)}=\sum_{i=1}^{k} a_{i} \partial^{-1} \Psi_{i}, \\
& \left(T^{(n, k)}\right)^{-1}=\sum_{j=0}^{k-n} \partial^{j} b_{j}+\sum_{j=1}^{n} \Phi_{j} \partial^{-1} b_{-j},
\end{aligned}
$$

which can be obtained in the same way as the case $n>k$. Then by using Table II

$$
\begin{aligned}
& T^{(n, k)}\left(\Phi_{i}\right)=0, T^{(n, k)}(1)=1, \\
& T^{(n, k)}=\alpha_{n-k} \partial^{n-k}+(\text { lower order terms })=\sum_{j=0}^{\infty}(-1)^{j} \sum_{i=1}^{k} a_{i} \Psi_{i}^{(j)} \partial^{-1-j}
\end{aligned}
$$

Therefore

$$
\left\{\begin{array}{l}
\sum_{i=1}^{k} a_{i} \int \Psi_{i} d x=1, \\
\sum_{i=1}^{k} a_{i} \int \Psi_{i} \Phi_{j} d x=0, j=n, n-1, \cdots, 1 \\
\sum_{i=1}^{k} a_{i} \Psi_{i}^{(j)}=0, j=0,1, \cdots, k-n-2
\end{array}\right.
$$


By solving this equation, (4.33) can be derived. And also by using

$$
\alpha_{n-k}=\sum_{i=1}^{k}(-1)^{-1+k-n} a_{i} \Psi_{i}^{(-1+k-n)}
$$

one can obtain

$$
\alpha_{n-k}=(-1)^{n} \frac{I W_{n, k}\left(\Phi_{n}, \Phi_{n-1}, \cdots, \Phi_{1} ; \Psi_{1}, \cdots, \Psi_{k}\right)}{I W_{n+1, k}\left(1, \Phi_{n}, \Phi_{n-1}, \cdots, \Phi_{1} ; \Psi_{1}, \cdots, \Psi_{k}\right)} .
$$

On the other hand, from (4.38), (4.41) and Table II,

$$
\begin{aligned}
& \left(\left(T^{(n, k)}\right)^{-1}\right)^{*}\left(\Psi_{i}\right)=0, i=1,2, \cdots, k \\
& b_{k-n}=\alpha_{n-k}^{-1}=(-1)^{n} \frac{I W_{n+1, k}\left(1, \Phi_{n}, \Phi_{n-1}, \cdots, \Phi_{1} ; \Psi_{1}, \cdots, \Psi_{k}\right)}{I W_{n, k}\left(\Phi_{n}, \Phi_{n-1}, \cdots, \Phi_{1} ; \Psi_{1}, \cdots, \Psi_{k}\right)} .
\end{aligned}
$$

Then

$$
\left\{\begin{array}{l}
\sum_{i=0}^{k-n}(-1)^{i} b_{i} \Psi_{j}^{(i)}-\sum_{i=1}^{n} b_{-i} \int \Phi_{i} \Psi_{j} d x=0, j=1,2, \cdots, k \\
b_{k-n}=(-1)^{n} \frac{I W_{n+1, k}\left(1, \Phi_{n}, \Phi_{n-1}, \cdots, \Phi_{1} ; \Psi_{1}, \cdots, \Psi_{k}\right)}{I W_{n, k}\left(\Phi_{n}, \Phi_{n-1}, \cdots, \Phi_{1} ; \Psi_{1}, \cdots, \Psi_{k}\right)}
\end{array}\right.
$$

which leads to (4.34).

\section{Applications of $T^{(n, k)}$}

In the last section, the explicit forms of $T^{(n, k)}$ have been obtained. In this section, we will investigate the applications of the above explicit formulas.

Firstly, let's consider the applications of $T^{(n, k)}$ on the eigenfunction and adjoint eigenfunction of the mKP hierarchy. We summarize the corresponding results in the next proposition.

Proposition 5.1. Under the successive gauge transformation $T^{(n, k)}$, the eigenfunction $\Phi$ (which is not proportional to $\Phi_{1}, \cdots, \Phi_{n}$ in $T^{(n, k)}$ ) and the adjoint eigenfunction $\Psi$ (which is not proportional to $\Psi_{1}, \cdots, \Psi_{k}$ in $T^{(n, k)}$ ) of the $m K P$ hierarchy will become into

- when $n>k$,

$$
\begin{aligned}
\Phi^{(n+k)}= & \frac{I W_{k, n+1}\left(\Psi_{k}, \Psi_{k-1}, \cdots, \Psi_{1} ; \Phi_{1}, \cdots, \Phi_{n}, \Phi\right)}{I W_{k, n+1}\left(\Psi_{k}, \Psi_{k-1}, \cdots, \Psi_{1} ; \Phi_{1}, \cdots, \Phi_{n}, 1\right)} \\
\Psi^{(n+k)}= & (-1)^{n+1} \frac{I W_{k, n+1}\left(\Psi_{k}, \Psi_{k-1}, \cdots, \Psi_{1} ; \Phi_{1}, \cdots, \Phi_{n}, 1\right)}{I W_{k, n}\left(\Psi_{k}, \Psi_{k-1}, \cdots, \Psi_{1} ; \Phi_{1}, \cdots, \Phi_{n}\right)^{2}} \\
& \times I W_{k+1, n}\left(\Psi, \Psi_{k}, \cdots, \Psi_{1} ; \Phi_{1}, \cdots, \Phi_{n}\right)
\end{aligned}
$$

- when $n=k$,

$$
\begin{aligned}
\Phi^{(n+n)}= & \frac{I W_{n, n+1}\left(\Psi_{n}, \Psi_{n-1}, \cdots, \Psi_{1} ; \Phi_{1}, \cdots, \Phi_{n}, \Phi\right)}{I W_{n, n+1}\left(\Psi_{n}, \Psi_{n-1}, \cdots, \Psi_{1} ; \Phi_{1}, \cdots, \Phi_{n}, 1\right)} \\
\Psi^{(n+n)}= & (-1)^{n+1} \frac{I W_{n, n+1}\left(\Psi_{n}, \Psi_{n-1}, \cdots, \Psi_{1} ; \Phi_{1}, \cdots, \Phi_{n}, 1\right)}{I W_{n, n}\left(\Psi_{n}, \Psi_{n-1}, \cdots, \Psi_{1} ; \Phi_{1}, \cdots, \Phi_{n}\right)^{2}} \\
& \times I W_{n, n+1}\left(\Phi_{1}, \cdots, \Phi_{n} ; \Psi, \Psi_{n}, \cdots, \Psi_{1}\right) .
\end{aligned}
$$


- when $n<k$

$$
\begin{aligned}
\Phi^{(n+k)}= & \frac{I W_{n+1, k}\left(\Phi, \Phi_{n}, \cdots, \Phi_{1} ; \Psi_{1}, \cdots, \Psi_{k}\right)}{I W_{n+1, k}\left(1, \Phi_{n}, \cdots, \Phi_{1} ; \Psi_{1}, \cdots, \Psi_{k}\right)}, \\
\Psi^{(n, k)}= & -\frac{I W_{n+1, k}\left(1, \Phi_{n}, \Phi_{n-1}, \cdots, \Phi_{1} ; \Psi_{1}, \cdots, \Psi_{k}\right)}{I W_{n, k}\left(\Phi_{n}, \Phi_{n-1}, \cdots, \Phi_{1} ; \Psi_{1}, \cdots, \Psi_{k}\right)^{2}} \\
& \times I W_{n, k+1}\left(\Phi_{n}, \cdots, \Phi_{1} ; \Psi, \Psi_{1}, \cdots, \Psi_{k}\right) .
\end{aligned}
$$

Proof. These results can be obtained by substituting (4.14), (4.15), (4.31), (4.32), (4.33) and (4.34) into the following expressions

$$
\Phi^{(n+k)}=T^{(n, k)}(\Phi), \quad \Psi^{(n+k)}=-\left(\left(T^{(n, k)}\right)^{-1}\right)^{*}(\Psi) .
$$

Proposition 5.2. Under the successive gauge transformation $T^{(n, k)}$, the tau functions $\tau_{0}$ and $\tau_{1}$ of the $m K P$ hierarchy will be changed into

- when $n \geq k$,

$$
\begin{aligned}
\tau_{0}^{(n+k)} & =\frac{I W_{k, n}\left(\Psi_{k}, \Psi_{k-1}, \cdots, \Psi_{1} ; \Phi_{1}, \cdots, \Phi_{n}\right) \tau_{1}^{n-k}}{\tau_{0}^{n-k-1}} \\
\tau_{1}^{(n+k)} & =\frac{I W_{k, n+1}\left(\Psi_{k}, \Psi_{k-1}, \cdots, \Psi_{1} ; \Phi_{1}, \cdots, \Phi_{n}, 1\right) \tau_{1}^{n-k+1}}{\tau_{0}^{n-k}}
\end{aligned}
$$

- when $n<k$

$$
\begin{aligned}
\tau_{0}^{(n+k)} & =\frac{I W_{n, k}\left(\Phi_{n}, \Phi_{n-1}, \cdots, \Phi_{1} ; \Psi_{1}, \cdots, \Psi_{k}\right) \tau_{1}^{n-k}}{\tau_{0}^{n-k-1}} \\
\tau_{1}^{(n+k)} & =\frac{(-1)^{n} I W_{n+1, k}\left(1, \Phi_{n}, \Phi_{n-1}, \cdots, \Phi_{1} ; \Psi_{1}, \cdots, \Psi_{k}\right) \tau_{1}^{n-k+1}}{\tau_{0}^{n-k}} .
\end{aligned}
$$

Proof. Here we only give the proof for the case of $n \geq k$, since the proof in the other case is almost the same. For this, assume

$$
T^{(n, k)}=\alpha_{n-k} \partial^{n-k}+\alpha_{n-k-1} \partial^{n-k-1}+\cdots .
$$

Then through (4.14) and (4.31), one can find

$$
\begin{aligned}
& \alpha_{n-k}=\frac{I W_{k, n}\left(\Psi_{k}, \Psi_{k-1}, \cdots, \Psi_{1} ; \Phi_{1}, \cdots, \Phi_{n}\right)}{I W_{k, n+1}\left(\Psi_{k}, \Psi_{k-1}, \cdots, \Psi_{1} ; \Phi_{1}, \cdots, \Phi_{n}, 1\right)} \\
& \alpha_{n-k-1}=-\frac{\partial_{x}\left(I W_{k, n}\left(\Psi_{k}, \Psi_{k-1}, \cdots, \Psi_{1} ; \Phi_{1}, \cdots, \Phi_{n}\right)\right)}{I W_{k, n+1}\left(\Psi_{k}, \Psi_{k-1}, \cdots, \Psi_{1} ; \Phi_{1}, \cdots, \Phi_{n}, 1\right)} .
\end{aligned}
$$

Further from the transformation

$$
Z^{(n+k)}=T^{(n, k)} Z^{(0)} \partial^{k-n}
$$


one can obtain

$$
\begin{aligned}
z_{0}^{(n+k)} & =\alpha_{n-k} z_{0}, \\
z_{1}^{(n+k)} & =\alpha_{n-k} z_{1}+(n-k) \alpha_{n-k} z_{0 x}+\alpha_{n-k-1} z_{0} .
\end{aligned}
$$

Then according to (5.14) and the relation between $\tau_{0}$ and $\tau_{1}$ given in (2.16), one can find

$$
\begin{aligned}
& -\partial_{x} \ln \left(\tau_{0}^{(n+k)}\right)=\frac{z_{1}^{(n+k)}}{z_{0}^{(n+k)}}=\frac{z_{1}}{z_{0}}+(n-k) \partial_{x} \ln \left(z_{0}\right)+\frac{\alpha_{n-k-1}}{\alpha_{n-k}} \\
& =-\partial_{x} \ln \left(\tau_{0}\right)+(n-k) \partial_{x} \ln \left(z_{0}\right)-\partial_{x} \ln \left(I W_{k, n}\left(\Psi_{k}, \Psi_{k-1}, \cdots, \Psi_{1} ; \Phi_{1}, \cdots, \Phi_{n}\right)\right),
\end{aligned}
$$

Therefore by (2.16), (5.14) and (5.16),

$$
\begin{aligned}
\tau_{0}^{(n+k)} & =\frac{I W_{k, n}\left(\Psi_{k}, \cdots, \Psi_{1} ; \Phi_{1}, \cdots, \Phi_{n}\right) \tau_{0}}{z_{0}^{n-k}}=\frac{I W_{k, n}\left(\Psi_{k}, \cdots, \Psi_{1} ; \Phi_{1}, \cdots, \Phi_{n}\right) \tau_{1}^{n-k}}{\tau_{0}^{n-k-1}} \\
\tau_{1}^{(n+k)} & =\frac{\tau_{0}^{(n+k)}}{z_{0}^{(n+k)}}=\frac{I W_{k, n+1}\left(\Psi_{k}, \Psi_{k-1}, \cdots, \Psi_{1} ; \Phi_{1}, \cdots, \Phi_{n}, 1\right) \tau_{1}^{n-k+1}}{\tau_{0}^{n-k}}
\end{aligned}
$$

In order to obtain the explicit examples of $\tau_{0}$ and $\tau_{1}$, we start from the zero solution, i.e., $L^{(0)}=$ $\partial$, which satisfies (2.1). One can easily find that in this case, the mKP hierarchy in KupershmidtKiso version coincides with the usual KP hierarchy. Denote

$$
\begin{aligned}
& \Phi_{i}^{(0)}=\exp \left(\xi\left(t, \alpha_{i}\right)\right)+a_{i} \exp \left(\xi\left(t, \beta_{i}\right)\right), i=1,2, \cdots, n, \\
& \Psi_{j}^{(0)}=\exp \left(-\xi\left(t, \gamma_{j}\right)\right)+b_{j} \exp \left(-\xi\left(t, \delta_{j}\right)\right), j=1,2, \cdots, k,
\end{aligned}
$$

where $\alpha_{i}, \beta_{i}, \gamma_{j}$ and $\delta_{j}$ are distinct complex numbers for different $i$ or $j$, and $a_{i}$ and $b_{j}$ are constants. Then one can easily check that $\Phi_{i}^{(0)}$ and $\Psi_{j}^{(0)}$ satisfy (2.17), and thus $\Phi_{i}^{(0)}$ and $\Psi_{j}^{(0)}$ are the corresponding (adjoint) eigenfunctions of the mKP hierarchy in Kupershmidt-Kiso version or the usual $\mathrm{KP}$ hierarchy with respect to $L^{(0)}$. And in particular, 1 is also the eigenfunction of the KP hierarchy corresponding to $L^{(0)}$. As for the tau functions in this case, one can easily find that

$$
\tau_{0}^{(0)}=1, \quad \tau_{1}^{(0)}=1
$$

Then according to Proposition 5.2 and [9], one has the following results

Proposition 5.3. Starting from the zero solution of the $m K P$ hierarchy, i.e., $L^{(0)}=\partial$, the tau pair $\left(\tau_{0}^{(n+k)}, \tau_{1}^{(n+k)}\right)$ of the $m K P$ hierarchy is showed as follows.

- when $n \geq k$,

$$
\begin{aligned}
& \tau_{0}^{(n+k)}=I W_{k, n}\left(\Psi_{k}^{(0)}, \Psi_{k-1}^{(0)}, \cdots, \Psi_{1}^{(0)} ; \Phi_{1}^{(0)}, \cdots, \Phi_{n}^{(0)}\right) \\
& \tau_{1}^{(n+k)}=I W_{k, n+1}\left(\Psi_{k}^{(0)}, \Psi_{k-1}^{(0)}, \cdots, \Psi_{1}^{(0)} ; \Phi_{1}^{(0)}, \cdots, \Phi_{n}^{(0)}, 1\right)
\end{aligned}
$$

- when $n<k$

$$
\begin{aligned}
\tau_{0}^{(n+k)} & =I W_{n, k}\left(\Phi_{n}^{(0)}, \Phi_{n-1}^{(0)}, \cdots, \Phi_{1}^{(0)} ; \Psi_{1}^{(0)}, \cdots, \Psi_{k}\right) \\
\tau_{1}^{(n+k)} & =(-1)^{n} I W_{n+1, k}\left(1, \Phi_{n}, \Phi_{n-1}, \cdots, \Phi_{1} ; \Psi_{1}, \cdots, \Psi_{k}\right) .
\end{aligned}
$$


Thus $\tau_{0}^{(n+k)}$ and $\tau_{1}^{(n+k)}$ can be viewed as the tau functions of the KP hierarchy.

Corollary 5.1. Starting from $L^{(0)}=\partial$, the tau pair $\left(\tau_{0}^{(n)}, \tau_{1}^{(n)}\right)$ of the $m K P$ hierarchy is listed below,

$$
\begin{aligned}
\tau_{0}^{(n)} & =W_{n}\left(\Phi_{1}^{(0)}, \cdots, \Phi_{n}^{(0)}\right), \\
\tau_{1}^{(n)} & =W_{n+1}\left(\Phi_{1}^{(0)}, \cdots, \Phi_{n}^{(0)}, 1\right) ;
\end{aligned}
$$

which are also the tau functions of the KP hierarchy.

Remark: the above results can help us to understand the tau pair $\left(\tau_{0}, \tau_{1}\right)$ of the mKP hierarchy in Kupershmidt-Kiso version.

\section{Conclusions and Discussions}

The elementary gauge transformation operators $T_{i}$ with $i=1,2,3$ (see Proposition 3.1) are discussed in Section 2. The actions of $T_{i}$ on the dressing operator, the (adjoint) eigenfunctions and the tau functions are presented in Proposition 3.2. Then the successive applications of gauge transformation operators $T_{i}$ are shown in Proposition 3.3. At the end of Section 2, $T_{i}$ are proved that they can not commute with each other (see (3.37)), which reflects that $T_{i}$ are not easy to carry out for the mKP hierarchy.

Therefore $T_{D}$ and $T_{I}$ (see (4.1) and (4.2)) are introduced in Section 3. And in particular, as far as I know, $T_{I}$ is introduced for the first time in the literature. After that, how the objects of the mKP hierarchy are transformed under $T_{D}$ and $T_{I}$ are listed in Proposition 4.1. And further based upon the results in Proposition 4.1, $T_{D}$ and $T_{I}$ are shown that they can commute with each other (see (4.5) -(4.7)) and hence they are more applicable in the mKP hierarchy than $T_{i}$ with $i=1,2,3$. Then the main part of Section 3 is devoted to the successive applications of $T_{D}$ and $T_{I}$. And the products of $n$ terms of $T_{D}$ and $k$-terms of $T_{I}$, denoted as $T^{(n, k)}$, are given in Proposition 4.2 for $n>k$, Proposition 4.3 for $n=k$ and Proposition 4.4 for $n<k$. At last, the actions of $T^{(n, k)}$ on the eigenfunction $\Phi$, the adjoint eigenfunction $\Psi$ and the tau functions $\tau_{0}$ and $\tau_{1}$ are presented in Proposition 5.1 and 5.2. Also the explicit forms of tau functions $\tau_{0}$ and $\tau_{1}$ are given in Proposition 5.3 and Corollary 5.1.

Compared with the KP hierarchy, there are several differences in the mKP hierarchy, which brings much difficulty to the study of the mKP hierarchy. 1) There is the second high order term in the Lax operator of the mKP hierarchy (see $u_{0}$ in (2.2)), which leads to the extra condition (see (4.4)) to determine $T^{(n, k)} .2$ ) The inverse of the conjugation of $T_{D}$ is not $T_{I}$ and vice versa. Therefore, the case $k>n$ must be discussed in the derivation of $T^{(n, k)}$. In fact, it is more difficult in the case $k>n$ than the cases $n>k$ and $n=k$, because it is very hard to determine the form of $T^{(n, k)}$ in the case $k>n$. 3) There are two tau functions $\tau_{0}$ and $\tau_{1}$ in the mKP hierarchy. Usually, it is more inconvenient to study the integrable systems with two tau functions than those with only one single tau function. Hence, additional methods must be developed in the discussion of the tau functions under the successive applications of $T_{D}$ and $T_{I}$ for the mKP hierarchy.

At last, the results in this paper are hoped to be generalized to the constrained mKP hierarchy. Also these results may be helpful to discuss the inner integrability of the mKP hierarchy such as the additional symmetries and the squared eigenfunction symmetries.

\section{Acknowledgments}

This work is supported by the Fundamental Research Funds for the Central Universities (Grant No. 2015QNA43). 


\section{References}

[1] H. Aratyn, E. Nissimov and S. Pacheva, Darboux-Backlund solutions of SL(p,q) KP-KdV hierarchies, constrained generalized Toda lattices, and two-matrix string model, Phys. Lett. A 201 (1995) 293-305.

[2] L. L. Chau, J. C. Shaw and H. C. Yen, Solving the KP hierarchy by gauge transformations, Commun. Math. Phys. 149 (1992) 263-278.

[3] L. L. Chau, J. C. Shaw and M. H. Tu, Solving the constrained KP hierarchy by gauge transformations, J. Math. Phys. 38 (1997) 4128-4137.

[4] J. P. Cheng and J. S. He, The applications of the gauge transformation for the BKP hierarchy, J. Math. Anal. Appl. 410 (2014) 989-1001.

[5] J. P. Cheng, J. Z. Wang and X. Y. Zhang, The gauge transformation of the $q$-deformed modified KP hierarchy, J. Nonlin. Math. Phys. 21 (2014) 533-542.

[6] Y. Cheng, Modifying the KP, the $n^{\text {th }}$ constrained KP hierarchies and their Hamiltonian structures, Commun. Math. Phys. 171 (1995) 661-682.

[7] E. Date, M. Jimbo, M. Kashiwara and T. Miwa, Transformation groups for soliton equations, in Nonlinear integrable systems - classical theory and quantum theory, edited by M. Jimbo and T. Miwa, (World Scientific, Singapore, 1983), 39-119.

[8] L. A. Dickey, Modified KP and discrete KP, Lett. Math. Phys. 48 (1999) 277-289.

[9] J. S. He, Y. S. Li and Y. Cheng, The determinant representation of the gauge transformation operators, Chin. Ann. Math. B 23 (2002) 475-486.

[10] J. S. He, Y. Cheng and R. A. Roemer, Solving bi-directional soliton equations in the KP hierarchy by gauge transformation, J. High Energy Phys. 03 (2006) 103.

[11] J. S. He, Y. H. Li and Y. Cheng, $q$-deformed KP hierarchy and $q$-Deformed constrained KP hierarchy, SIGMA 2 (2006), 060.

[12] J. S. He, Z. W. Wu and Y. Cheng, Gauge transformations for the constrained CKP and BKP hierarchies, J. Math. Phys. 48 (2007) 113519.

[13] R. Huang, T. Song and C. Z. Li, Gauge transformations of constrained discrete modified KP systems with self-consistent sources, Int. J. Geom. Methods Mod. Phys. 14 (2017) 1750052.

[14] M. Jimbo and T. Miwa, Solitons and infinite dimensional Lie algebras, Publ. RIMS, Kyoto Univ. 19 (1983) 943-1001.

[15] M. Kashiwara and T. Miwa, The $\tau$ function of the Kadomtsev-Petviashvili equation. Transfromation groups for soliton equations. I, Proc. Japan Acad. A 57 (1981) 342-347.

[16] K. Kiso, A remark on the commuting flows defined by Lax equations, Prog. Theo. Phys. 83 (1990) 1108-1125.

[17] B. Konopelchenko and W. Oevel, An $r$-matrix approach to nonstandard classes of integrable equations, Publ. RIMS, Kyoto Univ. 29 (1993) 581-666.

[18] B. A. Kupershmidt, Mathematics of dispersive water waves, Commun. Math. Phys. 99 (1985) 51-73.

[19] B. A. Kupershmidt, On the integrability of modified Lax equations, J. Phys. A: Math. Gen. 22 (1989) L993-L998.

[20] M. H. Li, J. P. Cheng and J. S. He, The gauge transformation of the constrained semi-discrete KP hierarchy, Mod. Phys. Lett. B 27 (2013) 1350043.

[21] M. H. Li, J. P. Cheng and J. S. He, The successive application of the gauge transformation for the modified semidiscrete KP hierarchy, Z. Naturforsch. A 71 (2016) 1093-1098.

[22] R. L. Lin, X. J. Liu and Y. B. Zeng, A new extended $q$-deformed KP hierarchy, J. Nonlin. Math. Phys. 15 (2008) 333-347.

[23] R. L. Lin, H. Peng and M. Manas, The $q$-deformed mKP hierarchy with self-consistent sources, Wronskian solutions and solitons, J. Phys. A: Math. Theor. 43 (2010) 434022.

[24] S. W. Liu, Y. Cheng and J. S. He, The determinant representation of the gauge transformation for the discrete KP hierarchy, Sci. China Math. 53 (2010) 1195-1206.

[25] J. J. C. Nimmo, Darboux transformations from reductions of the KP hierarchy, in Nonlinear Evolution Equations and Dynamical Systems, edited by V. G. Makhankov, A. R. Bishop, and D. D. Holm, (World Scientific, Singapore, 1995), 168-177. 
[26] W. Oevel and C. Rogers, Gauge transformations and reciprocal links in $2+1$ dimensions, Rev. Math. Phys. 5 (1993) 299-330.

[27] W. Oevel, Darboux theorems and Wronskian formulas for integrable system I: constrained KP flows, Physica A 195 (1993) 533-576.

[28] W. Oevel, Darboux transformations for integrable lattice systems, in Nonlinear Physics: theory and experiment, edited by E. Alfinito, L. Martina and F. Pempinelli, (World Scientific, Singapore, 1996), 233-240.

[29] J. C. Shaw and M. H. Tu, Miura and auto-Backlund transformations for the cKP and cmKP hierarchies, J. Math. Phys. 38 (1997) 5756-5773.

[30] J. C. Shaw and Y. C. Yen, Miura and Backlund transformations for hierarchies of integrable equations, Chin. J. Phys. 31 (1993) 709-719.

[31] T. Takebe, A note on the modified KP hierarchy and its (yet another) dispersionless limit, Lett. Math. Phys. 59 (2002) 157-172.

[32] T. Takebe and L. P. Teo, Coupled modified KP hierarchy and its dispersionless limit, SIGMA 2 (2006) 072.

[33] M. H. Tu, J. C. Shaw, and C. R. Lee, On Darboux - Backlund transformations for the $q$-deformed Korteweg - de Vries hierarchy, Lett. Math. Phys. 49 (1999) 33-45.

[34] R. Willox, I. Loris, C. R. Gilson, Binary Darboux transformations for constrained KP hierarchies, Inverse Problems 13 (1997) 849-865. 